\title{
Detection of Epileptic Seizure using Fast Adaptive Neuro Fuzzy Inference System
}

\author{
D. Karthika Renuka ${ }^{1}$ and L. Ashok Kumar ${ }^{2}$ \\ \{dkr.it@psgtech.ac.in ${ }^{1}$, lak.eee@psgtech.ac.in²\} \\ Sri Krishna College of Engineering and Technology, Coimbatore, Tamil Nadu, India ${ }^{1}$, Associate \\ Professor, Dept of IT, PSG College of Technology, Coimbatore, Tamil Nadu, India ${ }^{2}$, Professor, Dept of \\ EEE, PSG College of Technology, Coimbatore, Tamil Nadu, India ${ }^{3}$
}

\begin{abstract}
A diverse range of epilepsies suggest that its arousal may be caused by a variety of factors, but the whole process culminates in a common occurrence known as the seizure. The need for a precise and dependable seizure detector is critical, not only for a better understanding of seizures, but also as a benefit for medics who are fatigued by the prospect of picturing a continuous and massive long-term recording of seizures. The lack of an acceptable mix of methodologies, despite significant advancements in automated epilepsy diagnosis over the last decade, highlights the need for suitable seizure detection systems, which are not yet widely used. A wide variety of methods is being extensively explored, and every attempt is being made with the greatest amount of involvement in order to provide a strategy for seizure detection. Automatic seizure detection systems are primarily concerned with delivering a consistent algorithm by accumulating extensive knowledge of the dynamical characteristics of the signal as well as the clinical domains in which they operate. EEG signals are often clouded by the presence of artefacts, which makes it difficult to make an accurate diagnosis and conduct an accurate analysis. This step includes the implementation of a standard notch filter, followed by spatially restricted Independent Component Analysis and Wavelet denoising techniques in order to enhance the quality of EEG signals. Five different kinds of artefacts were identified and eliminated. For the delta, alpha, and beta bands, the power spectrum estimator (Welsh power spectrum estimator) was used to compute the frequency spectrum and the power. These data were utilised to create a feature vector, which was then used as an input to the next step of the process. The suggested classification algorithms are described in detail below. In order to identify the existence of a seizure in an EEG signal, a hybrid extreme machine learning is employed. It was decided to integrate the Modified Levenberg Marquardt algorithm (MLA) with the classifiers to decrease the time complexity.
\end{abstract}

Keywords: Electroencephalogram, Epilepsy, Spatial constraints, Extreme Machine Learning.

\section{Introduction}

Nervous system disorders include conditions affecting the brain, spinal cord, and nerves throughout the body that develop as a result of structural, biochemical, and electrical abnormalities. Many of the neurological diseases are quite common, with just a few of them being really rare. These diseases need a thorough neurological examination conducted by experienced neurologists and neuropsychologists in order to be properly diagnosed. It is 
estimated that there are about 600 recognised kinds of neurological disorders worldwide, ranging from migraines to epilepsy. The Global Burden of Disease (GBD) research is a joint effort of the World Health Organization (WHO), the World Bank, and the Harvard School of Public Health. According to the Globe Health Organization, these diseases and their severity have an impact on more than 1 billion people across the world, according to their size (World Health Organization, 2006). It is anticipated that this number would increase in line with the population growth, and these illnesses are becoming a significant danger to public health. Neurosurgeons can perform operations on these disorders if they are not treated with medication or through preventative measures. Preventative measures, lifestyle changes, physiotherapy or other therapy, neuro-rehabilitation, pain management, and medication are all options for treating these disorders. Confusion, loss of feeling, muscular weakness, paralysis, poor coordination, seizures, pain, and altered states of awareness are just a few of the symptoms associated with these diseases (http://www.medicalmarijuanainc.com/index.php/neurological-disorder). Researchers and policymakers believe that mortality data are the most important indicator of the severity of a disease, and that it is on the basis of these numbers that nations and organizations have started disease control efforts. The pain caused by diseases that are not deadly but may cause significant impairment, on the other hand, is grossly underestimated by such a number.

Most notable among India's significant accomplishments in the area of health are the decreasing trend in vaccine preventable diseases as a result of increased immunization coverage, and the sincere efforts being made to eradicate poliomyelitis via a nationwide pulse vaccination campaign. Extrapolating from the data, the prevalence rate of epilepsy is about 1.2 to 2 crore people in the general population [1]. Several free camps may be held with the aim of eradicating epilepsy or raising awareness about the illness among the general public. The primary motive for this thesis was a desire to relocate epileptic patients away from India by giving firsthand information to the subject (patient) via the use of a programmed to report whether or not seizures had befallen a certain individual. Taken together, these modest advances will add up to a huge leap forward, opening the door to broad use of the treatment arsenal for all other neurological disorders. The field of neuropsychiatry, a merged branch of medicine, has been regarded a single tree in the Western world for more than 2000 years, since it was first established. In the wake of some illnesses, conflicting ideas regarding their origin and pathophysiology emerged, and these views were the primary cause for the emergence of unfavorable sentiments among individuals, who responded with ridicule and incivility among themselves. Disdain among civilians should be eliminated by raising awareness among them via the implementation of health programmed and the education of civilians. It is true that social stigma is a source of distress. Certain neurological diseases were mistakenly categorized as psychopathological in origin, despite the fact that there is no clear distinction between disorders of psychopathology and neuropathology [2]. These disorders were the primary reasons of the patient's inharmonious attitude toward treatment. Because the words brain health and mental disease are no longer used interchangeably, it is anticipated that neurologists and psychiatrists would have a wide viewpoint in order to provide appropriate therapy. When dealing with confusion, a basic diagnostic instrument such as an automated seizure detection system may be very helpful in resolving it to a certain degree [3]. The detection of epileptic seizures is accomplished via the execution of a series of stages, which include signal collection, artefact removal, feature extraction, and classification. Already, various algorithms exist for each of these stages, but as of now, no research are focusing on the issue of how to combine the attractive techniques and create a more effective algorithm to achieve this goal. Therefore, selecting the most effective approach for each of these stages in 
order to achieve the greatest possible performance is very challenging. By creating an appropriate Automatic Seizure Detection System, this study effort tries to model a conglomeration of algorithms in each of the stages with the best and victorious result possible for each phase.

\section{Literature survey}

\section{EEG Signal}

Hans Berger (1873-1941) [4] made a significant contribution to the history of encephalography by finding the presence of human electroencephalograms (EEGs). His experiments began with a string galvanometer (1910), a model of the primordial type, and progressed to a smaller Edelmann model in 1912, before transitioning to a larger Edelmann model in 1924 and finally to a large Edelmann model in 1925. In 1926, he utilised a far more powerful galvanometer, as shown in Figure 2.2, to record the brain activity of his first patient's brain activity. In 1929, he established annals by recording human EEG on photographic paper for a period of one to three minutes using a one-channel bipolar technique with frontooccipital leads and a duration of one to three minutes. He was the first to uncover the alpha rhythm and to identify it as the primary component of the electroencephalogram (EEG). He was deeply involved in the research of cerebral localization, and he worked closely with colleagues on the localization of brain tumours. Specifically, he looked at the relationship between mental processes and changes in EEG signals. In 1930, he was the first to do research on the recording of sleep spindles, which are the pinnacle of EEG synchronisation during sleep. He was also the first to report on the effects of hypoxia, which is defined as a lack of oxygen to the brain.

In their work on artefact removal, Muhammad et al. (2011) used spatial restrictions on the ICA to achieve their results. ICAs with spatial constraints were suggested to extract artifactonly independent components, which was then followed by Wavelet Denoising (WD) to eliminate brain activity from the retrieved independent components. The recovered artifactonly signals are projected back and removed from the EEG data to provide a clean EEG that may be used for further analysis and processing. It was the work done by Muhammad et al. that sparked the idea for phase I of this research. The emphasis is on obtaining artifact-only data, which come after a lengthy process that requires hauling the artefact information all the way back to the computer and subtracting it from EEG signals. Because of this, it has an effect on the processor's time complexity and memory usage. In order to prevent a lengthy operation, some changes have been considered during the preprocessing stage of our investigation, according to the researchers. In contrast to artifactual information, only cerebral information is retrieved. Wavelet Denoising is then used in order to eliminate any artefact information from the image. By combining FastICA, InfomaxICA, and ExtendedInfomaxICA, it is possible to create three distinct geographically restricted algorithms: Spatially Constrained FastICA, Spatially Constrained InfomaxICA, and Spatially Constrained Extended Infomax ICA. Daubechies performed the Wavelet Transformation, which was followed by Otsu and fuzzy shrink thresholding to complete the process. This study effort was based on the work done [5], and the fuzzy shrink thresholding method was used in this investigation. The work carried out by them consists in the use of a wavelet-based fuzzy denoising method for the denoising of 81e 84 single and multi-channel pictures, respectively. The intra-scale dependence of the wavelet co-efficient was used to describe the fuzzy characteristic. This fuzzy feature was 
utilised to differentiate between noisy and noiseless coefficients, as well as to improve the information contained in wavelet coefficients during the shrinking process. A fuzzy membership function was employed to reduce the wavelet coefficients depending on the fuzzy feature, and the result was a smaller wavelet coefficient. The same approach that was utilised for picture denoising was applied to the denoising of EEG signals as previously described.

AzadehBastani and colleagues [6] conducted a research in which they compared the Walsh Hadamard transform and the Fourier analysis of electroencephalogram (EEG) data. Their research shown that the performance of the Walsh Hadamard transform is more successful in extracting the characteristics of the signals from EEG signals during the sleep phases. For real-time signal processing, it is recommended that WHT be used rather than Fourier transform (FT), due to the lower number of operations, simplicity of implementation by digital circuits, and speed of the WHT. Fourier transform (FT) requires a higher volume of operations, which makes it less suitable for real-time signal processing. In light of the assessment of the comparative study between WHT and FT, it was heuristically concluded that the Fast Walsh Hadamard Transform (FWHT) may be a viable alternative to the Fast Fourier Transform (FFT). This study's second phase uses FWHT for the extraction of EEG signals from the bands in the bands. According to Ravish et al. [7], an analysis was carried out based on the extraction of parameter values such as power distribution in different phases of EEG time series and spectral power in various frequency bands of EEG. Seizures were detected using the power distribution in the alpha and delta bands, which were calculated. In accordance with this philosophy, the PSD values are computed in each of the bands derived from the FWHT data. For the following phase categorization, the statistical fluctuations (minimum and maximum PSD values, mean and standard deviation, among other things) are used as input. Classification was performed in the third phase of the study, which was carried out using the Hybrid Extreme Learning Machine (HELM) with Modified LevenbergMarquardt (MLM) algorithm, which uses the Analytical Hierarchical Process (AHP) method with minor modifications in the algorithm, which was proposed [8]. Yuedong Song et al. [9] developed an automated seizure detection system 85 based on sample entropy and ELM, which was successfully deployed. An implementation of PSD and HELM was a projection of the research work done by Songs and colleagues. Amal Mohamed and colleagues, while developing their new algorithm, placed a strong emphasis on the learning speed of FNN. He developed his method primarily to replace the sluggish gradient-based learning algorithm and the tediousness required in tuning repetition of parameters in these learning algorithms. His approach was adopted primarily for these reasons [10]. The AHP technique is used to choose the input weights and hidden biases, the ELM algorithm is used to calculate the output weights analytically, and the LM algorithm is used to train the network, among other things. Using his model, we were able to improve the pace of our EEG-based epilepsy research, which was accomplished by including yet another change to the LM algorithm. It was decided to use ANFIS as a comparison study classification since it is based on the Neuro Fuzzy model. A slight change was performed by applying MLM in the backward pass instead of Gradient Descent (GD), resulting in FANFIS, in order to conduct comparative research (Fast ANFIS).

On the basis of the motivation and prevailing factors behind the disease, a research problem is developed with the following strategy: "To design an Automatic Seizure Detection system that would efficiently operate at the extremes of all three axes simultaneously, namely high accuracy, high scalability, and ease of implementation with its utility." Utilizing signal processing and soft computing methods, it is possible to achieve the aforementioned strategy by developing and implementing an Automated Seizure Detection System using 40 
Electroencephalogram (ASDEEG), which is used to detect seizures. The following were the goals that were established for the development and implementation of ASDEEG [11].

- To create and develop preprocessing methods that minimise the artefacts that hide the underlying cerebral brain activity and allow for more accurate interpretation of the data.

To design and develop a feature extraction method to discover patterns that will be used to assist in the following stages of the ASDEEG process.

- To design and build a classification phase that makes use of the characteristics collected to determine if a seizure is present or not.

to conduct a performance study of the three suggested methods and to compare their results with those of already in use systems.

\section{Materials and Methodology}

The visual inspection of electroencephalographic or electrocorticographic recordings may quickly identify the different kinds of seizures and their spread throughout the nervous system. This procedure, however, is excruciatingly slow and error-prone owing to the weariness of the participants. Efforts to automate the process of identifying seizures by probing through different dynamic characteristics of EEG waveforms have been made in recent years. Monitoring and analysis of EEG signals are used to determine when an epileptic seizure is about to begin. By determining the appropriate action, it is possible to decrease morbidity and mortality associated with seizures. Neurologists use visual analysis to assess large quantities of multi-channel EEG data in order to achieve a certain objective. In order to achieve a quick and balanced EEG analysis, it is necessary to automate the process of identifying seizures

\subsection{Acquisition of Input Signals}

A significant advancement in the technology of EEG amplifiers and recording devices has occurred in the last 20 years. Computer-based digitization and storage have mostly replaced paper-based recording. The EEG of the scalp provides preliminary indications of neuronal synchronisation in the underlying cortical activity. The electrode placement technique utilised by EEG sensors for data collection is the 10/20 system, which is a widely accepted method of electrode insertion. Through the use of this method, a connection between the position of an electrode and the underlying region of the cerebral cortex may be established. These signals are saved in a digital format to be retrieved later. The amplitude and frequency specification of signals are two characteristics that distinguish them. It has been discovered that the amplitude of EEGs recorded by intra cerebral or subdural electrodes is greater than that of EEGs recorded by scalp electrodes. When scalp electrodes are used, amplitudes in the range of $20 \mathrm{~V}$ to $100 \mathrm{~V}$ are recorded, and amplitudes in the range of $100 \mathrm{~V}$ to $2 \mathrm{MV}$ are recorded when depth electrodes are used, respectively [12]. The EEG has a spectral bandwidth ranging from $0.5 \mathrm{~Hz}$ to about $500 \mathrm{~Hz}$. In order to record data appropriate for timefrequency analysis, it is necessary to take certain key factors into account while configuring the amplifier and filter settings for EEG data collection. One of the most pressing issues is the need to sample EEG signals at a higher rate in order to prevent signal aliasing due to frequency aliasing. Alignment errors are caused primarily by the consequences of under sampling. Alignment errors result in the misrepresentation of a high-frequency signal as a low-frequency signal. The Nyquist rate is the lowest sampling rate that may be used to prevent aliasing while still maintaining quality. Even while typical EEG collection software imposes a higher requirement, such as a sampling rate that is four times the greatest frequency of interest, it is twice as fast as the highest 
frequency of interest in this case [13]. The raw EEG signal is made up of two sets of data for 160 individuals, one of which corresponds to disease and the other which corresponds to normalcy. The dataset includes 16 channel recordings for 160 patients, with each recording lasting 10 seconds in duration. Samples of the data were taken at a rate of 256 samples per second throughout the experiment. This means that a single data set has 4096 samples overall, which when multiplied by 160 patients results in a dataset consisting of 65,53,600 samples over 10 seconds in a 16-channel recording from that data set. This diagram depicts the taxonomy of the signal acquisition system Figure 1.

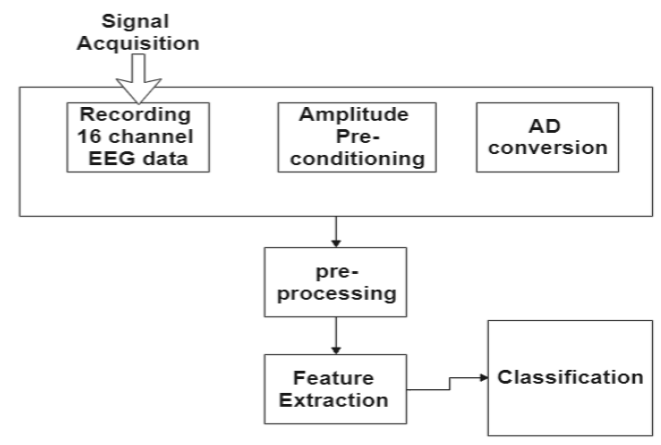

Figure. 1 represents the taxonomy of the signal acquisition system

Signal preconditioning is used in the signal acquisition system because it improves the quality of the signals by establishing predetermined voltage or current for the purpose of establishing proper operating conditions. Bias and amplitude preconditioning are used because they improve the signal to noise ratio and the strength of the neural signal, both of which are important. It is essential to note at this point that the noise produced by state-of-the-art amplifiers is very tiny and insignificant when compared to the EEG signals being recorded and processed [14]. ADCs are used to convert the amplification signals into serial output signals after they have been stored in Sample and Hold (S\&H) circuits. An analogue multiplexer is used to scan the S\&H outputs, and the signals are converted sequentially to create serial output signals.

\subsection{Pre-Processing}

Pre-processing is a collection of methods for removing artefacts from images. Artifacts from inside the building as well as from outside are taken into account.

The term "Feature Extraction" refers to a set of techniques for effectively extracting spectral frequency bands and deriving a feature based on the power spectral density in each of these bands, as well as other terms [15].

C. Classification - this includes methods and processes that are used to distinguish between seizure and non-seizure signals. Each of the aforementioned stages is dealt with individually, and the methods that are used in each of these phases are improved in order to achieve effective manoeuvring. The fact that these phases are integrated, meaning that the output of one phase serves as an input to the next, is also significant. Because of the benefits gained via artefact removal in the first phase, there is a positive effect on the final phase. It is suggested that preprocessing be included as an optional argument in the ASDEEG framework, which may be toggled on and off to highlight the actual necessity for artefact removal.

\subsection{Feature extraction using Independent Component Analysis (ICA)}


ICA is an implementation of Blind Source Separation (BSS), and it is used to create source independence between two or more sources. As a result, it has acquired widespread acceptance in the area of biomedical signal processing [15]. Figure 2 depicts the taxonomy of the International Classification of Animals (ICA)

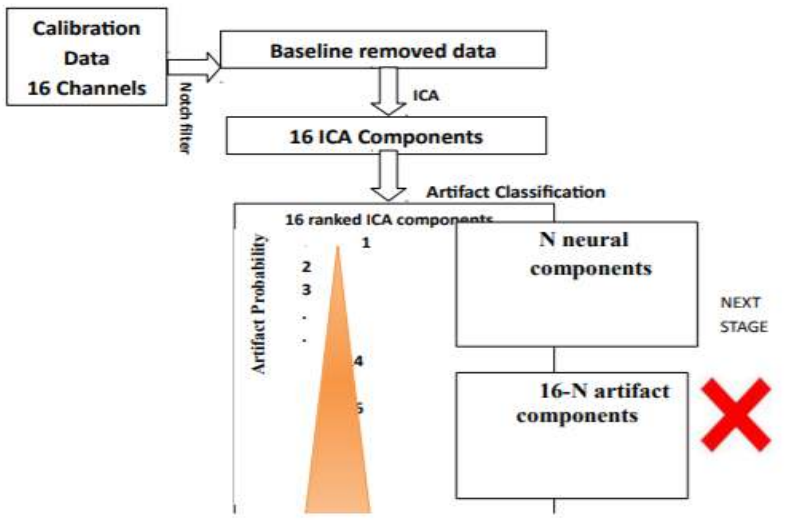

Figure 2 Taxonomy of classification

When separating multi-channel signals into their basic underlying components, it is called demodulation. The ICA model is strongly linked with a number of key assumptions, including: I In the case of underlying observable sources, statistical independence is assumed to exist. If the observed linear mixture ' $\mathrm{m}$ ' is more than the number of independent components ' $n$,' then the observed linear mixture ' $\mathrm{m}$ ' is greater than the number of independent components ' $n$.' iii) The signal from each sensor has a different mixing ratio of the independent components than the signal from the other sensors. Artifact Capacity for a bility Illustration of the classification of ICA $\mathrm{N}$ neural components.Components of artefacts number 16-N ICA, according to Hyvarinen et al. (2000), is "a statistical signal processing method that models a collection of observations, $\mathrm{x}$, with an instantaneous linear mixing of independent latent variable '.s'

$\mathbf{x}(t)=\mathbf{A s}(t)+n s(t)$

in which'ns'represents additive noise over a time period of ' $t$ ' Let'x'denote the random vectors whose elements are the mixes $\mathrm{x} 1, \ldots, \mathrm{x} \mathrm{n}$, and let's' denote the random vectors whose components are the components $\mathrm{s} t, \ldots, \mathrm{s}$ a, respectively. In this case, let $\mathrm{A}$ be the matrix holding the elements'aij.'

In this case, the issue is to identify both the matrix'A'and the independent components's' that have a known value, namely the measured variables'x ', using the known value as the starting point. With the assumption that component's i' is independent and must have a nonGaussian distribution, the following postulation is made: The mixing matrix has the shape of a square. Because the ICA model is used to estimate sources, it may be expressed in a more straightforward manner as

$\mathrm{s}=\mathrm{Wx}$

where' $\mathrm{W}$ 'denotes the inverse of the estimated matrix 'A', and where there are many ways for determining independence, and each of them involves the use of a distinct algorithm or set of algorithms. In terms of ICA algorithms, there are two major groups (Haykin, 2009). The minimization of mutual information is ingrained in the design of certain algorithms, whereas the maximising of non-Gaussianity is ingrained in the design of others. It is possible to get various unmixing matrices by using different algorithms. The non-Gaussianity of the data is 
utilised to determine the independence in this research. A technique for separating the components, based on the premise that each underlying source does not follow a normal distribution, involves causing each of the sources to deviate significantly from the normal distribution.

3.4 Otsu's Thresholding

This technique, which is named after its creator, Nobuyuki Otsu, is a bimodal algorithm that is used to solve optimization problems (1979). This technique iterates over all of the potential threshold values and produces a measure that includes both sides of the threshold, i.e. the peak and the valley of the signal, as well as the middle of the threshold. We want to determine the value at which the total of peak and valley spreads is at its smallest, and this is the threshold value. By reducing the within-class variation or increasing the between-class variance, the Otsu's approach determines the appropriate threshold to use.

A signal is made up of $\mathrm{N}$ values, each having a level ranging from 1 to $\mathrm{L}$. The number of values of level $\mathrm{I}$ is represented by the function $\mathrm{f} i$, with the probability given as

$p_{i}=f_{i} / N$

As a result, the values are divided into two classes, $C 1$ with levels [1...t] and $\mathrm{C} 2$ with levels $[\mathrm{t}+1, \ldots, \mathrm{L}]$, which are then further divided into subclasses. The level probability distributions for the two classes are then calculated as follows:

$$
\begin{aligned}
& \quad \omega_{1}|t|=\sum_{i=1}^{t} p_{i} \\
& \text { and } \\
& \omega_{2}|t|=\sum_{i=t+1}^{L} p_{i}
\end{aligned}
$$

Also, the means for classes $\mathrm{C}_{1}$ and $\mathrm{C}_{2}$ are

$$
\text { And } \mu_{1}=\sum_{i=1}^{t} i p_{i} / \omega_{1}|t| \text { (6) }
$$

$\mu_{1}=\sum_{i=r+1}^{L} i p_{i} / \omega_{2} \mid t$

Let $\mu_{T}$ be the mean intensity for the whole values. It is easy to show that

$\omega_{1} \mu_{1}+\omega_{2} \mu_{2}=\mu_{T}$

$\omega_{1}+\omega_{2}=1$

Using discriminate analysis, between-class variance of the thresholded data, and is denoted as

$$
\sigma_{n}^{2}=\omega_{1}\left(\mu_{1}-\mu_{T}\right)^{2}+\omega_{\zeta}\left(\mu_{2}-\mu_{\tau}\right)^{2}
$$

For bi-level thresholding, the optimal threshold $t^{*}$ is selected so that the betweenclass variance $\sigma_{B}^{2}$ is maximized, which is denoted as

$$
t^{2}=\operatorname{Arg} \operatorname{Max}\left|\sigma_{B}^{2}\right| t \mid .1 \leq t<L
$$

The introduction of fuzzy set theory by Zadeh(1965) opened the door for the capture of uncertainty and ambiguity in complex systems, which is frequently ignored in practise. The theory of fuzzy sets is an extension of the idea of classical sets. Fuzzy systems are systems that are either knowledge-based or rule-based in nature. It is the fuzzy IF-THEN rules that constitute the basis of the knowledge base, which are at the core of every fuzzy system. A fuzzy set is an enumeration of items that has a membership function that has a continuum grade of membership. It is distinguished by a membership function, which gives each object a membership grade ranging between zero and one on a scale from zero to one. The method employed in this research is based on the idea of windows and incorporates the following factors, which serve as a foundation for computing the fuzzy feature: It is possible that signal components are present if both the primary value of the window (ws,d(i, j)) and the average value of the neighbour (xs,d(i and j)) coefficients are sufficiently high.

- Even if the average value of the neighbour is greater than the major coefficient, the main coefficients are still considered signal components. 
- If the main and average value of neighbour are both small enough, the coefficients are considered to be noisy components.

- The letters 'S' and 'd' stand for scaling and orientation, respectively.

\section{Results and Discussion}

\subsection{Fast Adaptive Neuro Fuzzy Inference System (FANFIS)}

Classification Model is a kind of classification system that categorises objects into groups. Uncertain systems are poorly represented by traditional mathematical methods, which makes them unsuitable for system modelling. The use of fuzzy rules in a fuzzy inference system allows it to mimic the requirements imposed by human knowledge. According to the authors, the overall goal of this study is to propose a novel architecture dubbed FANFIS, which employs an effective technique for adjusting the membership functions in order to reduce the output error while simultaneously maximising speed. One of the criteria of FANFIS is the construction of an adequate set of fuzzy if-then rules to adjust membership functions in order to execute the specified input-output operation, which is also one of the requirements of FANFIS. FANFIS is made up of five different functional components. - A rule-based block consisting of fuzzy if-then rules is one kind of block.

- A database block consisting of membership functions for fuzzy sets is used in this example. An inference operation for fuzzy rules is performed by a decision block, which is part of the decision block.

- A fuzzification interface unit, which performs the task of converting crisp input values into degrees of match with linguistic values by changing them into degrees of match with linguistic values.

- A defuzzification interface unit, which performs the opposite function of the preceding interface unit. It converts fuzzier findings into more precise output values.

\subsection{Performance Parameters}

The most essential stage in every research project is the assessment of the performance of the techniques that have been adopted, improved, and created. Three stages are involved in the proposed ASDEEG system's operation, and each of these phases needs a distinct set of performance criteria for assessment. The primary goal is to find techniques in each of the stages by comparing them to the ways that were used as a yardstick in the previous phase. All of the stages are then brought together to create a complete system that performs better than the sum of its parts. Varying researchers place different emphasis on different factors while doing their study.

\subsection{Mean Square Error (MSE)}

When comparing two signals, the Mean Square Error (MSE) is used to determine the degree of similarity/fidelity (or conversely, the level of error/distortion) between them. It is based on an assumption that one of the signals is an exact replica of the pristine original, while the other has been distorted or contaminated by errors.

$$
\mathrm{MSE}=\frac{\sum\left[I_{1}(m, n)-I_{2}(m, n)\right]^{2}}{M^{*} N}
$$

The numbers $\mathrm{M}$ and $\mathrm{N}$ in the above equation indicate the number of rows and columns in the supplied input signal. When comparing signals, the Peak Signal-to-Noise Ratio (PSNR), also known as the signal-to-noise ratio (SNR), defines the relationship between the highest potential strength of a signal and the power of corrupting noise that has an adverse effect on 
the conformity of its representation. When describing sound levels, the logarithmic decibel scale is often used. It is simple to calculate using the MSE.

$$
\text { PSNR }=10 \log _{10}\left(\frac{M a x^{2}}{M S E}\right)
$$

The highest value of the EEG signal is denoted by the symbol Max in the preceding equation. The greater the value of the PSNR, the higher the quality of the signal is considered to be.

Pre-processing time is a measurement that is used to estimate the amount of time that will be needed to improve the signals.

\subsection{Power Spectral Density (PSD)}

The PSD is a measure of the power or variance of a time series that is distributed with regard to frequency distribution. It refers to the amount of power that may be delivered per unit of frequency. When it came to the Classification phase, the PSD values were utilised to train the network.

$$
\mathrm{PSD}=\frac{1}{\mathrm{k}} \sum_{\mathrm{m}=0}^{\mathrm{K}-1} \mathrm{Px}_{m}, \mathrm{M}\left(\omega_{\mathrm{K}}\right)
$$

In the preceding equation, Px $\mathrm{m}$ represents the periodogram computed using a Welch estimate of the input signal of length $\mathrm{m}$, and $\mathrm{K}$ defines the window size; in the following equation, Px m signifies the periodogram calculated using a Welch estimate of the input signal of length $\mathrm{m}$.

Specificity

Specificity is a statistical metric that is used to assess the performance of classification algorithms and systems. True negative rate is a term that refers to the percentage of negatives that are properly recognised, and it is also used to quantify this proportion. It is most often stated as a percentage of the total.

Specificity $=\frac{\text { Number of true negative decisions }}{\text { Number of actual negative cases }}(15)$

Sensitivity

Similarly, sensitivity is a statistical metric that is used to show the percentage of positives that are really recognised properly. True positive rate is another term for this statistic. It is most often stated as a percentage of the total.

Sensitivity $=\frac{\text { Number of true positive decisions }}{\text { Number of actual positive cases }}(16)$

Accuracy

As defined by the International Organization for Standardization, accuracy is the degree to which a measured value corresponds to a true or recognised value. Accuracy may be defined as the degree to which something is accurate. The accuracy of a classifier is what determines its final performance. It is most often stated as a percentage of the total.

$$
\text { Accuracy }=\frac{\text { Number of correct decisions }}{\text { Total number of cases }}(17)
$$

TABLE 1 Mean Square Error for Spatially Constrained ICAs

\begin{tabular}{|l|l|l|l|l|l|}
\hline Techniques & $\begin{array}{l}\text { Electrical } \\
\text { Artifact }\end{array}$ & $\begin{array}{l}\text { Eye Ball } \\
\text { Movement } \\
\text { Artifact }\end{array}$ & $\begin{array}{l}\text { Eye Blink } \\
\text { Artifact }\end{array}$ & $\begin{array}{l}\text { Jaw } \\
\text { Clenching } \\
\text { Artifact }\end{array}$ & $\begin{array}{l}\text { Spit } \\
\text { Swallowing } \\
\text { Artifact }\end{array}$ \\
\hline $\begin{array}{l}\text { SC } \\
\text { InfomaxICA }\end{array}$ & 10.4790 & 10.4790 & 7.1112 & 14.6513 & 12.0170 \\
\hline
\end{tabular}




\begin{tabular}{|l|l|l|l|l|l|}
\hline $\begin{array}{l}\text { SC } \\
\text { Extended } \\
\text { InfomaxICA }\end{array}$ & 1.8660 & 5.8995 & 1.6601 & 2.0230 & 2.0230 \\
\hline SC FastICA & 1.0001 & 1.0007 & 1.0015 & 1.0036 & 1.0015 \\
\hline
\end{tabular}

According to the table, the geographically restricted FastICA method beats the other Spatially Constrained ICA algorithms, as shown by the fact that it has the lowest MSE values of all the algorithms presented. According to this estimate, the next best performer in terms of MSE is geographically constrained Extended InfomaxICA, which has MSE values that are lower than those found for spatially restricted InfomaxICA. It can be shown that SCFastICA beats SCExtendedInfomax ICA by an insignificant factor of 1.86 when it comes to electrical artefact. and SCInfomaxICA by a factor of 10.5. It outperforms SCExtendedInfomax ICA by a factor of 5.89 for Eye Ball movement artifact and SCInfomaxICA by a factor of 10.5. It outperforms SCExtendedInfomax ICA by a very negligible factor of 1.66 for eye blink artifact and SCInfomaxICA by a factor of 7. It outperforms SCExtendedInfomax ICA by a factor of 2 for Jaw Clenching artifact and SCInfomaxICA by a factor of 6 . Itoutperforms SCExtendedInfomax ICA by a factor of 2 for Spit Swallowing artifact and SCInfomaxICA by a factor of 12 .

An examination of the suggested spatially restricted algorithms with signal denoising, based on the MSE estimate, is then carried out with regard to different artefact interferences, using the MSE estimator as a benchmark. Data from spatially restricted InfomaxICA +DWT with Otsu and Fuzzy shrink thresholding are shown in Table 4.2, which also contains the analytical findings. The fuzzy Shrink thresholding outperforms the Otsu thresholding in terms of performance. When it comes to fuzzy shrink thresholding, the S shaped curve, followed by Sigmoid, B Splines, and Bell shaped curve membership functions outperform the $Z$ shaped, triangular, and Gaussian curves in terms of performance.

For the purpose of evaluating the overall performance of these algorithms in relation to each of the artefacts, the data for MSE, PSNR, and execution time are displayed. Using the estimator MSE with regard to Electrical Artifact, Figure 4.2 depicts the overall performance of algorithms with respect to the estimate MSE.

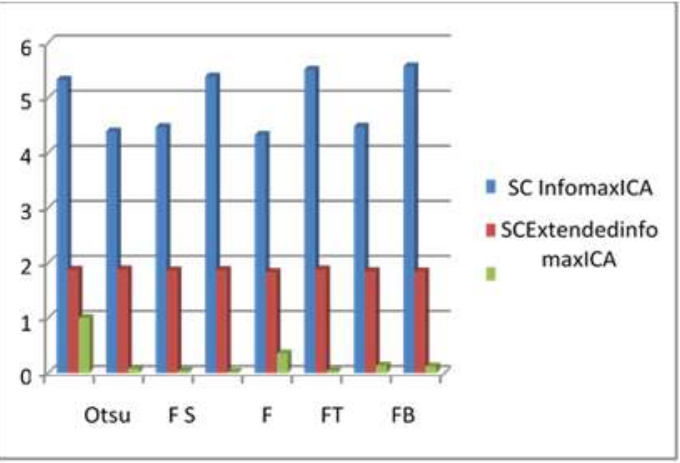

Figure 3 MSE of Electrical Artifact

With regard to Eyeball Movement Artifact, Figure 4.3 depicts the plot for overall performance of algorithms based on mean square error (MSE) values. 


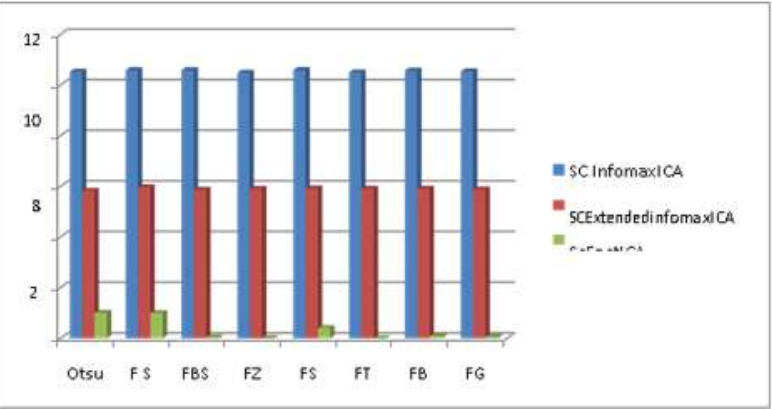

Figure 4 MSE of Eye Ball Movement Artifact

Figure 4Jaw Clenching Artifact embedded with Eye Ball movement artifact
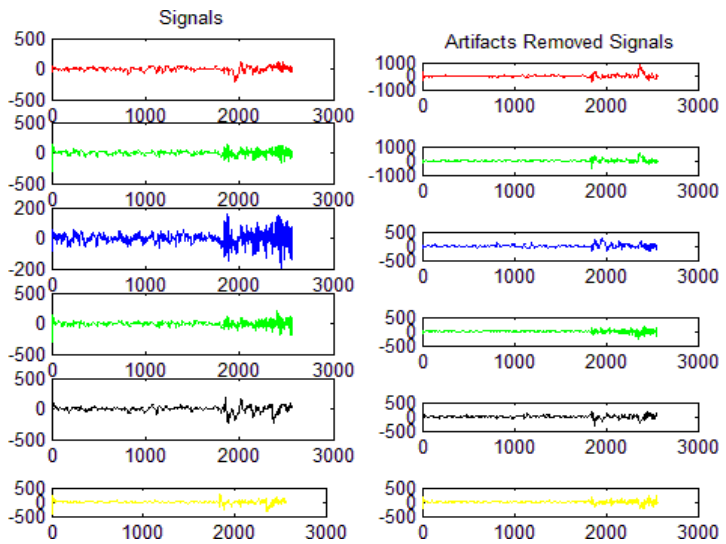

Figure 5 Removal of Jaw Clenching Artifact embedded with Eye Ball movement artifact using SCInfomaxICA

From the various results discussed it is clearly revealed that, spatially constrained fastICA with fuzzy thresholding performed well throughout the whole process of artifact removal. This was approved from the estimators MSE, PSNR, and time taken.

\section{Discussion}

An Epileptic Seizure Detection system based on a new statistical parameter Sample entropy for feature extraction. This was the only input feature vector fed as input to the classifiers BPNN and ELM. The data set used by Song is a benchmark dataset and the one adopted in this research work is a real time dataset obtained from local hospitals of Coimbatore. The EEG recording adopted by Song comprised of only 5 volunteers using a single channel of electrode, but the one used in this research work comprised of 160 subjects with 16 electrode channels. A classification accuracy of $95.67 \%$ was achievedin an assimilation of Sample entropy and ELM. This proposed system aimed in further improving the performance of the above work by making several alterations over the system proposed by 
SoFigure 4SCInfomaxICA is used to remove a jaw clenching artefact that was embedded with an eye ball movement artefact.

Following an examination of all of the data, it is apparent that spatially restricted fastICA with fuzzy thresholding outperformed all other approaches throughout the whole process of artefact removal. This was accepted by the estimators MSE, PSNR, and the amount of time it took.It was inspired by Yedong Song (2010), who proposed an Epileptic Seizure Detection system that used a novel statistical metric Sample entropy for feature extraction and was published in IEEE Transactions on Information Technology. This was the sole feature vector that was used as input to the classifiers BPNN and ELM, and it was the only one used. The data set utilised by Song is a benchmark dataset, while the data set used in this study effort is a real-time dataset collected from local hospitals in Coimbatore and used as the basis for this investigation. In contrast to Song's EEG recording, which consisted of just 5 volunteers utilising a single channel of electrodes, the EEG recording utilised in this study effort consisted of 160 participants using a total of 16 channels of electrodes. An assimilation of Sample entropy and ELM resulted in a classification accuracy of 95.67 percent in terms of classification accuracy. By introducing various modifications to the system provided by Song, this suggested system intended to further improve the performance of the aforementioned work. It was the use of methods for artefact removal that was the first modification made to the system developed by Song et al., and it had a significant effect on increasing the accuracy rate of the proposed system. The performance of the suggested winning classifier, the HELM, was also ascribed to the feature values derived from the FWHT and PSD. The accuracy of the winning classifier, which is comprised of a collection of algorithms, has been determined to be 99.56 percent.ng. Adopting techniques for artifact removal was the first alteration made to the system implemented by Song et al, which had an impact in augmenting the accuracy rate of the proposed system. The FWHT + PSD based feature values also attributed to the performance of the proposed winning classifier, the HELM. This compendium of algorithm which makes up the winning classifier has achieved an accuracy of $99.56 \%$.

\section{References}

[1] Abdulhamit Subasi, Ahmet Alkan, Etem Koklukaya and Kemal Kiymk, M. (2005) Wavelet neural network classification of EEG signals by using AR model with MLE preprocessing, Neural Networks, Vol.18, No.17, Pp. 985-997.

[2] Abdulhamit Subasi (2007) EEG signal classification using wavelet feature extraction and a mixture of expert model, Expert Systems with Applications, Vol.32, No.4, Pp. 1084-1093.

[3] Alessandro, D., Esteller, R., Vachtsevanos, G., Hinson, A., Echauz, J. and Litt, B. (2003) Epileptic Seizure Prediction Using Hybrid Feature Selection over Multiple Intracranial EEG Electrode Contacts: A Report of Four Patients, IEEE Transactions on Biomedical Engineering, Vol.50, No.5, Pp.603-615.

[4] Alkan, A. and Kiymik, M.K. (2006) Comparison of Auto Regressive and Welch methods in epileptic seizure detection, Journal of Medical Systems, Vol.30, No.6, Pp.413-419

[5] Babb, T.L., Mariani, E. and Crandall, P.H. (1974) An electronic circuit for detection of EEG seizures recorded with implanted electrodes, Vol. 37, Pp. 305-308.

[6] Bai, D., Qiu,T. and Li,X.(2007) The sample entropy and its application in EEG based epilepsy detection, Vol.24, No.1, Pp.200-205.

[7] Balli, T. and Palaniappan, R. (2010) Classification of biological signals using linear and nonlinear features, Physiological Measurement, Vol.31, Pp. 903. 
[8] Gotman, J. and Gloor, P. (1976) Automatic recognition and quantification of interictal epileptic activity in human scalp EEG, Journal of Electroencephalography and Clinical Neurophysiology, Vol. 41, Pp. 513- 529.

[9] Gotman, J., Gloor, P. and Schaul, N. (1978) Comparison of traditional reading of the EEG and automatic recognition of interictal epileptic activity, Journal of Electroencephalography and Clinical Neurophysiology, Vol. 44, Pp. 48-60

[10] Hagan, M.T. and Menhaj, M. (1994) Training feedforward networks with Marquardt algorithm, IEEE Transactions on Neural Networks, Vol.5,Pp. 983-993.

[11] Hart, Y. M. (2004). Management of newly diagnosed epilepsy. In S. Shorvon, D. R.Fish, E. Percucca \& W. E. Dodson (Eds.), The Treatment of Epilepsy (2nd ed., pp. 161-173). Oxford, England: Balckwell Science.

[12] D. S. Vijayan, A. Leema Rose, S. Arvindan, J. Revathy, C. Amuthadevi, "Automation systems in smart buildings: a review", Journal of Ambient Intelligence and Humanized Computing https://doi.org/10.1007/s12652-020-02666-9

[13] Loomis, A.L., Harvey, E.N. and Hobart, G.A.I. (1937) Cerebral states during sleep. As studied by human brain potentials, Journal Experimental Psychology, Vol. 21, Pp.127-44.

[14] D. S. Vijayan, A. Mohan, J. J. Daniel, V. Gokulnath, B. Saravanan, and P. D. Kumar, "Experimental Investigation on the Ecofriendly External Wrapping of Glass Fiber Reinforced Polymer in Concrete Columns," vol. 2021, 2021.

[15] Tumor Cell Morphology, Comparative Oncology,Baba AI, Câtoi C,Bucharest: The Publishing House of the Romanian Academy; 2007. http://www.ncbi.nlm.nih. gov/books/NBK9553, Access Date:22-03-2013. 